\title{
Colloidal Characteristics of Molybdenum Blue Nanoparticles Dispersion for Catalytic Applications ${ }^{+}$
}

\author{
Natalia Gavrilova *, Maria Myachina, Ksenia Poluboyarinova, Ekaterina Novaeva and \\ Victor Nazarov
}

Citation: Gavrilova, N.; Myachina, M.; Poluboyarinova, K.; Novaeva, E.; Nazarov, V. Colloidal Characteristics of Molybdenum Blue Nanoparticles Dispersion for Catalytic

Applications. Mater. Proc. 2021, 4, 24. https://doi.org/10.3390/

IOCN2020-07893

Academic Editors: Ana María Díez-Pascual, Antonio Di Bartolomeo and Guanying Chen

Published: 11 November 2020

Publisher's Note: MDPI stays neutral with regard to jurisdictional claims in published maps and institutional affiliations.

Copyright: $@ 2021$ by the authors. Licensee MDPI, Basel, Switzerland. This article is an open access article distributed under the terms and conditions of the Creative Commons Attribution (CC BY) license (http://creativecommons.org/licenses/by/4.0/).
Department of Colloid Chemistry, Faculty of Natural Sciences, D. Mendeleev University of Chemical Technology of Russia, Miusskaya sq., 9, 125047 Moscow, Russia; mmyachina@muctr.ru (M.M.); amalieleon@gmail.com (K.P.); kat.novaeva2015@yandex.ru (E.N.); nazarov@muctr.ru (V.N.)

* Correspondence: ngavrilova@muctr.ru

+ Presented at the 2nd International Online-Conference on Nanomaterials, 15-30 November 2020; Available online: https://iocn2020.sciforum.net/.

\begin{abstract}
The nanosized clusters of molybdenum blues and their monodispersity make them possible to consider as promising precursors for molybdenum carbide preparation. For the synthesis of supported catalysts using sols (dispersions of nanoparticles), it is necessary to know their main colloidal-chemical properties (electro-surface characteristics, rheological properties and the conditions of aggregative stability). This paper presents the results of a study of the colloidal-chemical properties of molybdenum blue, the dispersed phase of which is represented by toroidal particles of the Mo154-x family. It was found that aggregate stable sols exist in the range of $0.8<\mathrm{pH}<2.0$. In this range, molybdenum blue particles are negatively charged, and the electrokinetic potential does not exceed $30 \mathrm{mV}$. Molybdenum blues have high aggregate stability and can be concentrated to a high concentration of the dispersed phase (20-30 wt \%); at a concentration more than $30 \mathrm{wt} \%$, a transition of the sol into a gel is observed. In a wide range of concentrations, molybdenum blues are Newtonian liquids, and the viscosity mainly depends on the concentration of the dispersed phase. The results obtained can be used as a basis for the development of a sol-gel method of supported catalysts based on molybdenumblue.
\end{abstract}

Keywords: molybdenum blue; molybdenum oxide nanocluster; aggregativestability

\section{Introduction}

In recent years, the development of catalysts based on transition metal carbides has become increasingly important in chemical technology, since transition metal carbides are similar in catalytic properties to noble metals. One of these promising objects is molybdenum carbide $\mathrm{Mo}_{2} \mathrm{C}$. Molybdenum carbide shows high efficiency in the reactions of hydrazine decomposition [1], cellulose conversion [2] and hydrogenation [3-5], as well as in reactions involving hydrogen (methane conversion [6-8], water gas shift reaction [9,10], isomerization [11], etc.).

The most widely used heterogeneous catalysts are supported catalysts. To synthesize supported catalysts, the sol-gel method can be used, which allows the variation of the properties of the catalyst in a wide range. The sol-gel method of preparing $\mathrm{Mo}_{2} \mathrm{C}$ catalysts can be carried out using molybdenum blue (dispersions of molybdenum oxide clusters) obtained with organic reducing agents; this avoids an additional stage of catalyst activation [12,13]. To develop the main stages of the sol-gel method, knowledge of the main colloidal-chemical properties of dispersions, such as electro-surface and rheological characteristics, and conditions of aggregate stability is needed. 
The purpose of this work is to study the aggregative stability and electro-surface and rheological characteristics of dispersions of molybdenum blue prepared with ascorbic acid.

\section{Materials and Methods}

\subsection{Materials}

Ammonium heptamolybdate $\left(\mathrm{NH}_{4}\right)_{6} \mathrm{Mo}_{7} \mathrm{O}_{24} 44 \mathrm{H}_{2} \mathrm{O}$, ascorbic acid $\mathrm{C}_{6} \mathrm{H}_{8} \mathrm{O}_{6}$, and hydrochloric acid $\mathrm{HCl}$ were used for the molybdenum blue dispersion preparation. All reagents were purchased in CT Lantan, Moscow, Russia.

\subsection{Synthesis of Molybdenum Blue Dispersion}

Ascorbic acid and hydrochloric acid were added to a solution of ammonium heptamolybdate $(0.07 \mathrm{M})$ with vigorous stirring. The appearance of a bright blue color of the dispersion indicated the formation of molybdenum blue. The molar ratios $[\mathrm{R}] /[\mathrm{Mo}]=1.0$, molar ratios $[\mathrm{H}] /[\mathrm{Mo}]=0.8$ were selected on the basis of previous studies [14]. It is these conditions that make it possible to synthesize aggregatively and chemically stable molybdenum blue.

\subsection{Molybdenum Blue Dispersion Characterization}

Dynamic light scattering (Photocor Compact-Z instrument, Photocor LLC, Moscow, Russia) was used to characterize the particle size distribution of molybdenum blue.

Optical density and electronic absorption spectra were recorded using a Leki SS2110 UV scanning spectrophotometer (MEDIORA OY, Helsinki, Finland).

The electrophoretic mobility of hydrosol particles was evaluated using Photocor Compact-Z (LLC Photocor, Moscow, Russia). Electrokinetic potential was calculated using the Henry equation [15]:

$$
\zeta=\frac{3 \eta u_{\mathrm{ef}}}{2 \varepsilon \varepsilon_{0}} \cdot \frac{1}{\mathrm{f}_{1}(\kappa r)}
$$

where Uef is electrophoretic mobility, $\varepsilon$ is the dielectric constant of the medium, $\varepsilon_{0}$ is the electric constant, $\eta$ is the viscosity, $\mathrm{r}$ is the radius of particles, and $\kappa$ is the reciprocal of the Debye length. We calculated the function $\mathrm{f} 1(\mathrm{\kappa r})$ using the relation [16]:

$$
\mathrm{f}_{1}(\kappa \mathrm{r})=1+\frac{1}{2}\left[1+\left(\frac{2,5}{\kappa r[1+2 \exp (-\kappa \mathrm{r})]}\right)\right]^{-3}
$$

The rheological properties of molybdenum blue dispersion were studied by a Brookfield LV DV II viscosimeter (Brookfield Engineering Laboratories Inc, Middleboro MA, USA) using a ULA adapter. The measurements were carried out at $20 \pm 0.1{ }^{\circ} \mathrm{C}$. Brookfield TC-502 thermostat (Brookfield Engineering Laboratories Inc, USA) was used to maintain a constant temperature.

\section{Results}

\section{1. $p H$ Region of Aggregative Stability}

The $\mathrm{pH}$ range in which dispersions retain aggregate and chemical stability is one of the most important properties of sols as dispersed systems. To determine this region, molybdenum blue samples were prepared which differed in $\mathrm{pH}$. Optical density at the maximum absorption $(760 \mathrm{~nm})$ corresponds to the concentration of particles in the dispersed phase. The constancy of its value, as well as the hydrodynamic radius, indicates the stability of the dispersions.

Figure 1 shows the dependence of optical density on the $\mathrm{pH}$ value of the dispersion medium 1 and 7 days after synthesis. 


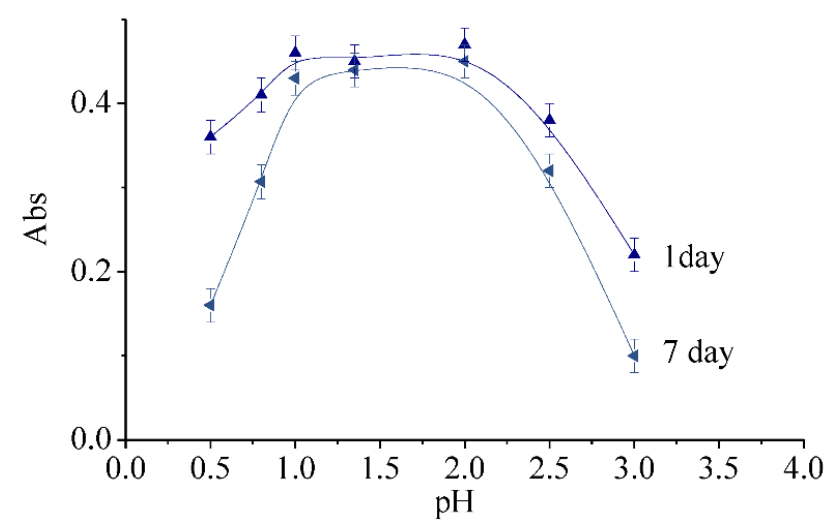

Figure 1. The dependence of optical density of molybdenum blue on the $\mathrm{pH}$ value of the dispersion.

At $\mathrm{pH}$ values of 0.8 or less, a drop in the optical density of dispersions is observed. At thesame time, the coagulation of particles and precipitation is observed. In the region of $\mathrm{pH} 2.5$ and more, a decrease in optical density is also observed, but this is due to a different reason. With increasing $\mathrm{pH}$, the destruction of molybdenum oxide clusters occurs. Molybdenum blue particles dissolve to form molybdate ions $\left(\mathrm{Mo}^{\mathrm{VI}}\right)$.

Thus, molybdenum blues synthesized using ascorbic acid are aggregatively and chemically stable in a narrow $\mathrm{pH}$ range from 0.8 to 2.0 .

\subsection{Electrokinetic Potential}

The electrokinetic potential was used as a characteristic of the electrosurface properties of dispersions. Molybdenum blues with different $\mathrm{pH}$ values are shown in Figure 2.

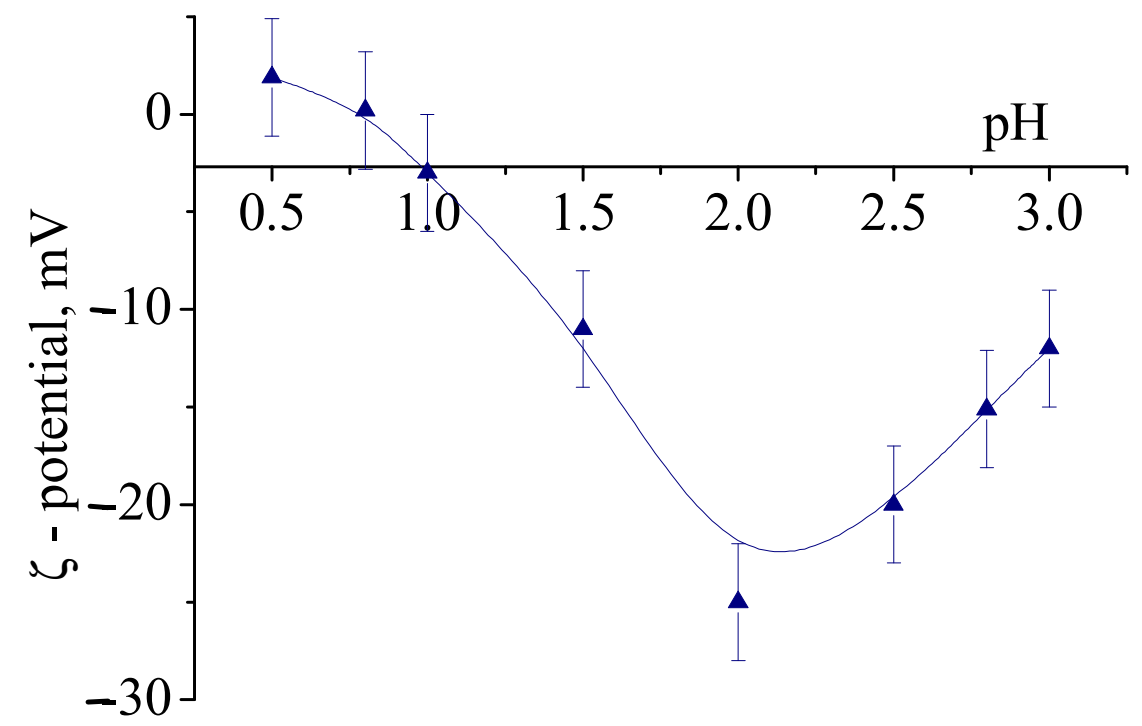

Figure 2. Dependence of the electrokinetic potential of molybdenum blue on the $\mathrm{pH}$ of the dispersion medium.

As shown in Figure 2 data, in the $\mathrm{pH}$ region from 3.0-0.8 particles are negatively charged. The maximum (in absolute value) value of the electrokinetic potential $(-28 \mathrm{mV})$ corresponds to the $\mathrm{pH}$ range of $\sim 2.0-2.5$.

In the range of $\mathrm{pH}$ values from 2.0 to 0.8 , the absolute value of the $\zeta$-potential decreases, accompanied by a change in sign at a $\mathrm{pH}$ value of 0.5 . The obtained value is close to the position of the isoelectric point for molybdenum trioxide $\mathrm{MoO}_{3}$, which, according to the literature, is in the range of $\mathrm{pH}$ values from 0.5 to 2.0 [17]. 


\subsection{Rheological Properties}

Rheological properties are of great importance for the synthesis of deposited materials by the sol-gel method. To determine the rheological properties of molybdenum blue, a series of samples with different concentrations of particles and different $\mathrm{pH}$ values (in the region of aggregate stability of systems) were prepared.

Experiments have shown that the dispersions of molybdenum blue synthesized using ascorbic acid are Newtonian liquids. Figure 3 shows the flow curves and the dependence of the viscosity on the shear stress for the molybdenum blue sample with $\mathrm{pH}=1.5$.

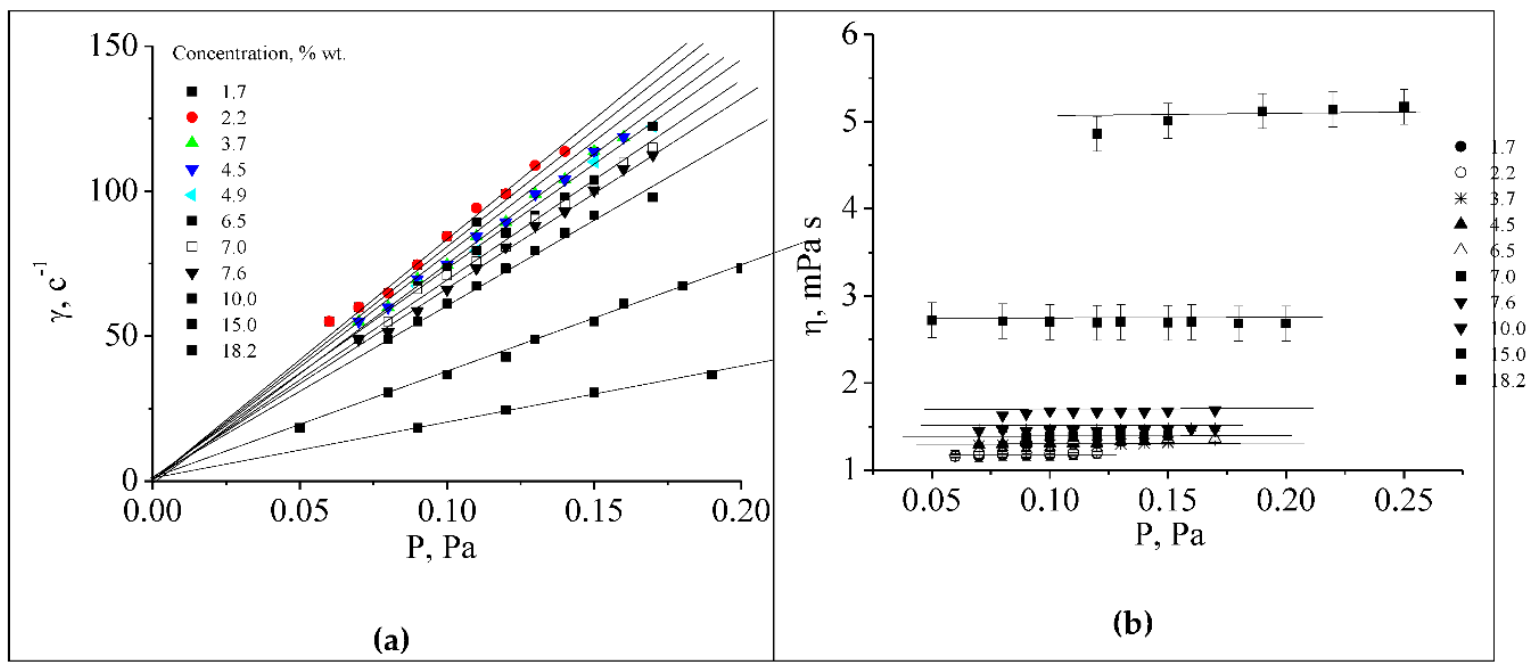

Figure 3. (a): Flow curves and (b): dependence of viscosity on load for molybdenum blue with different concentrations of particles $(\mathrm{pH}=1.5)$.

From the data obtained, it follows that the flow curves in the studied concentration range are linear dependences, which indicates that these hydrosols are Newtonian liquids with low viscosity. Figure 4 and the same data are presented in coordinates of specific viscosity on the volume fraction of the dispersed phase. When the volume fraction is more than 0.025 , a deviation from the linear dependence of the specific viscosity is observed. This is due to the appearance of electro-viscous effects, which are manifested in the dispersions of molybdenum blue on the particles of which there is a double electric layer. When constrained conditions arise, the diffuse parts of the DEL overlap, which leads to a sharper increase in viscosity with increasing particle concentration.

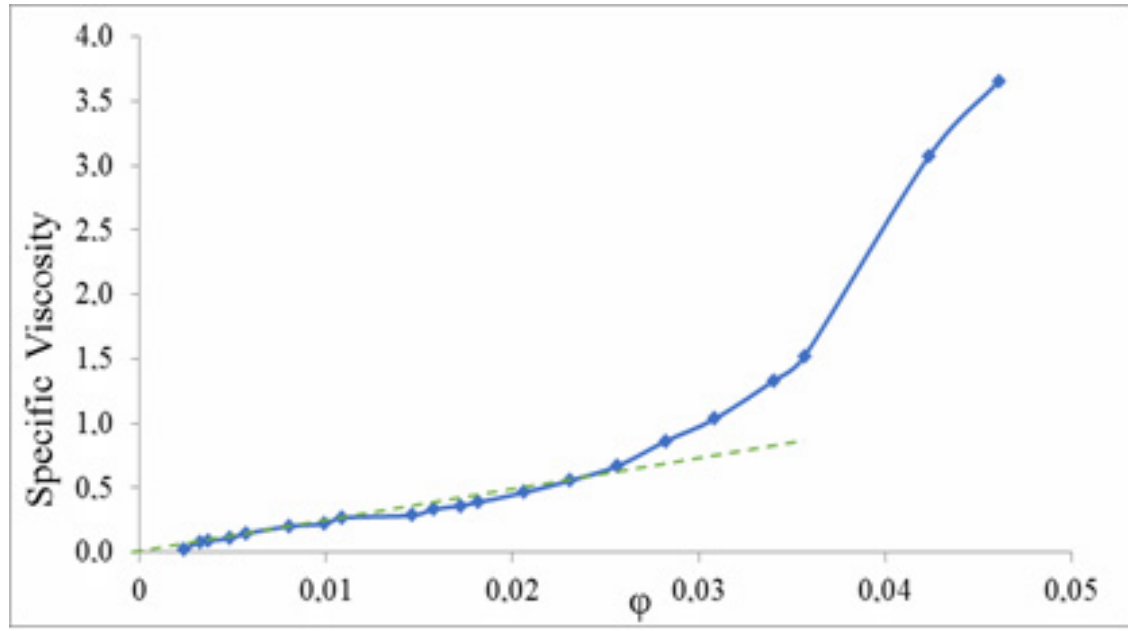

Figure 4. The dependence of the specific viscosity on the concentration of molybdenum blue $(\mathrm{pH}=1.5)$. 


\section{Discussion}

The presented results of the study of the colloidal-chemical properties of molybdenum blueare a continuation of the work [14], in which the features of the synthesis of molybdenum using ascorbic acid were considered. This dispersion is of undoubted interest, since it allows one step to synthesize highly dispersed molybdenum carbide [13], which is a catalyst for many reactions. The data on the basic colloidal-chemical characteristics are very important in the transition from the synthesis of powdered to supported $\mathrm{Mo}_{2} \mathrm{C}$ catalysts.

Author Contributions: Conceptualization, N.G., M.M., V.N.; methodology, N.G., M.M.; investigation, E.N., K.P., M.M., N.G.; resources, N.G.; writing-original draft preparation, M.M., N.G.; writing-review and editing, M.M., N.G., V.N.; visualization, M.M., N.G.; supervision, N.G., V.N. All authors have read and agreed to the published version of the manuscript.

Funding: This research was funded by D. Mendeleev University of Chemical Technology, grant number 032-2020.

Institutional Review Board Statement: Not applicable.

Informed Consent Statement: Not applicable.

Data Availability Statement: The data presented in this study are available on request from the corresponding author.

Conflicts of Interest: The authors declare no conflict ofinterest.

\section{References}

1. Chen, X.; Zhang, T.; Ying, P.; Zheng, M.; Wu, W.; Xia, L.; Li, T.; Wang, X.; Li, C. A novel catalyst for hydrazine decomposition: Molybdenum carbide supported on $\gamma-\mathrm{Al}_{2} \mathrm{O}_{3}$. Chem. Commun. 2002, 288-289, doi:10.1039/B109400A.

2. Ji, N.; Zhang, T.; Zheng, M.; Wang, A.; Wang, H.; Wang, X.; Shu, Y.; Stottlemyer, A.L.; Chen, J.G. Catalytic conversion of cellulose into ethylene glycol over supported carbide catalysts. Catal Today 2009, 147, 77-85.

3. Qin, Y.; Chen, P.; Duan, J.; Han, J.; Lou, H.; Zheng, X.; Hong, H. Carbon nanofibers supported molybdenum carbide catalysts for hydrodeoxygenation of vegetable oils. RSC Adv. 2013, 3, 17458-17491.

4. Maméde, A.S.; Giraudon, J.-M.; Löfberg, A.; Leclercq, L.; Leclercq, G. Hydrogenation of toluene over $\beta$-Mo2 $\mathrm{C}$ in the presence of thiophene. Appl. Catal. A Gen. 2002, 227, 73.

5. Ardakani, S.J.; Liu, X.; Smith, K.J. Hydrogenation and ring opening of naphthalene on bulk and supported Mo2 C catalysts. Appl. Catal. A Gen. 2007, 324, 9.

6. Christofoletti, T.; Assaf, J.; Assaf, E. Methane steam reforming on supported and nonsupported molybdenum carbides. Chem. Eng. J. 2005, 106, 97-103.

7. La Mont, D.C.; Thomson, W.J. Dry reforming kinetics over a bulk molybdenum carbide catalyst. Chem. Eng. Sci. 2005, 60, 35533559 .

8. Tominaga, H.; Nagai, M. Theoretical study of methane reforming on molybdenum carbide. Appl. Catal. A Gen. 2007, 328, 35-42.

9. Liu, P.; Rodriguez, J.A. Water-gas-shift reaction on molybdenum carbide surfaces: Essential role of the oxycarbide. J. Phys. Chem. B 2006, 110, 19418-19425.

10. Tominaga, H.; Nagai, M. Density functional theory of water-gas shift reaction on molybdenum carbide. J. Phys. Chem. B 2005, 109, 20415-20423.

11. Han, J.; Duan, J.; Chen, P.; Lou, H.; Zheng, X.; Hong, H. Nanostructured molybdenum carbides supported on carbon nanotubes as efficient catalysts for one-step hydrodeoxygenation and isomerization of vegetable oils. Green Chem. 2011, 13, $2561-2568$.

12. Gavrilova, N.N.; Nazarov, V.V.; Skudin, V.V. Synthesis of Membrane Catalysts Based on Mo2C. Kinet. Catal. 2015, 56, 670-680.

13. Gavrilova, N.; Dyakonov, V.; Myachina, M.; Nazarov, V.; Skudin, V. Synthesis of Mo2C by Thermal Decomposition of Molybdenum Blue Nanoparticles. Nanomaterials 2020, 10, 2053, doi:10.3390/nano10102053.

14. Gavrilova, N.; Myachina, M.; Harlamova, D.; Nazarov, V. Synthesis of Molybdenum Blue Dispersions Using Ascorbic Acid as Reducing Agent. Colloids Interfaces 2020, 4, 24, doi:10.3390/colloids4020024.

15. Henry, D.C.; Lapworth, A. The cataphoresis of suspended particles. Part I-The equation of cataphoresis. Proc. R. Soc. Lond. Ser. A 1931, 133, 106-129.

16. Ohshima, H. A simple expression for Henry's function for the retardation effect in electrophoresis of spherical colloidal particles. J. Colloid Interface Sci. 1994, 168, 269-271, doi:10.1006/jcis.1994.1419.

17. Kosmulski, M. Surface Charging and Points of Zero Charge; CRC Press: Boca Raton, FL, USA, 2009; p. 1092. 